\author{
Marquette University \\ e-Publications@Marquette
}

College of Nursing Faculty Research and

Publications

Nursing, College of

2020

\title{
Clinical Nurses' Perspectives on Discharge Practice Changes from Participating in a Translational Research Study
}

\author{
Kathleen L. Bobay \\ Loyola University Chicago \\ Regina Conway-Philips \\ Loyola University Chicago \\ Ronda G. Hughes \\ University of South Carolina \\ Linda L. Costa \\ University of Maryland - Baltimore \\ Sarah J. Bahr \\ Marquette University
}

See next page for additional authors

Follow this and additional works at: https://epublications.marquette.edu/nursing_fac

Part of the Nursing Commons

\section{Recommended Citation}

Bobay, Kathleen L.; Conway-Philips, Regina; Hughes, Ronda G.; Costa, Linda L.; Bahr, Sarah J.; Siclovan, Danielle M.; Nuccio, Susan A.; and Weiss, Marianne E., "Clinical Nurses' Perspectives on Discharge Practice Changes from Participating in a Translational Research Study" (2020). College of Nursing Faculty Research and Publications. 810.

https://epublications.marquette.edu/nursing_fac/810 
Authors

Kathleen L. Bobay, Regina Conway-Philips, Ronda G. Hughes, Linda L. Costa, Sarah J. Bahr, Danielle M. Siclovan, Susan A. Nuccio, and Marianne E. Weiss 
Marquette University

e-Publications@Marquette

\section{Nursing Faculty Research and Publications/College of Nursing}

This paper is NOT THE PUBLISHED VERSION; but the author's final, peer-reviewed manuscript. The published version may be accessed by following the link in the citation below.

Journal of Nursing Management, Early Access (October 6, 2020). DOI. This article is (C) Wiley and permission has been granted for this version to appear in e-Publications@Marquette. Wiley does not grant permission for this article to be further copied/distributed or hosted elsewhere without the express permission from Wiley.

\section{Clinical Nurses' Perspectives on Discharge Practice Changes from Participating in A Translational Research Study}

Kathleen L. Bobay

Marcella Niehoff School of Nursing, Loyola University Chicago, Chicago, IL Regina Conway-Phillips

Marcella Niehoff School of Nursing, Loyola University Chicago, Chicago, IL

Ronda G. Hughes

Center for Nursing Leadership, University of South Carolina College of Nursing, Columbia, SC Linda Costa

University of Maryland School of Nursing, Baltimore, MD

Sarah J. Bahr

Marquette University College of Nursing, Milwaukee, WI

Danielle Siclovan

Risk Management Services, Froedtert \& Medical College of Wisconsin, Milwaukee, WI

Susan Nuccio

Marquette University College of Nursing, Milwaukee, WI 


\title{
Marianne Weiss
}

Marquette University College of Nursing, Milwaukee, WI

\begin{abstract}
Aim

To describe clinical nurses' experiences with practice change associated with participation in a multisite nursing translational research study implementing new protocols for hospital discharge readiness assessment.

Background

Nurses' participation in translational research studies provides an opportunity to evaluate how implementation of new nursing interventions affects care processes within a local context. These insights can provide information that leads to successful adoption and sustainability of the intervention.
\end{abstract}

\section{Methods}

Semi-structured focus groups from 30 of 33 participating study hospitals lead by team nurse researchers.

\section{Results}

Nurses reported improved and earlier awareness of patients' discharge needs, changes in discharge practices, greater patient/family involvement in discharge, synergy and enhanced discharge processes, and implementation challenges. Participating nurses related the benefits of participation in nursing research.

\section{Conclusion}

Participation in a unit-level translational research project was a successful strategy for engaging nurses in practice change to improve hospital discharge.

Implications for Nursing Management

Leading unit-based implementation of a structured discharge readiness assessment including nurse assessment and patient self-assessment encourages earlier awareness of patients' discharge needs, improved patient assessment and greater patient/family involvement in discharge preparation. Integrating discharge readiness assessments into existing discharge care promotes communication between health team members that facilitates a timely, coordinated discharge.

\section{INTRODUCTION}

Translating research into practice has often been a difficult and time-consuming process, with research taking many years for full adoption (Weiss et al., 2018). Only a small proportion of nursing research ever reaches practice (Munro \& Savel, 2016). Proactive strategies for facilitating the transfer of research and shortening the timeline to adoption in nursing practice are needed. Studying how implementation of new nursing interventions affects nursing care practices within a local context can 
provide information that leads to successful adoption and sustainability of the intervention (Rabin \& Brownson, 2018).

The goal of this study was to describe clinical nurses' experiences with participating in a multi-site unitlevel nursing, translational research study evaluating the effectiveness of a structured discharge readiness assessment protocol implemented as a standard nursing practice (Weiss et al., 2019). Specifically, the aims were to (a) describe practice changes in unit and individual nurses' discharge processes over the course of the study; and (b) describe nurses' perceptions about participating in a unit-level nursing research study.

\section{BACKGROUND}

Nursing translational research involves testing the effectiveness of evidence-based protocols (EBP) within the real-world context of clinical practice and evaluation of the contextual factors that facilitate or inhibit the effectiveness of the study intervention (Weiss et al., 2018). Understanding context is important for learning why implementation of best practice does or does not work in a given setting (Titler, 2018).

The participation of clinical nurses in nursing research that tests the impact of new innovations or EBP can increase the knowledge transfer through experiential implementation and facilitate integration of new practices (Glasgow et al., 2014). Providing clear guidelines for implementation while allowing individualization for each nursing unit's preferences leads to successful and more consistent practice change. Careful planning and communication are essential to success (Melnyk et al., 2018). Integration into workflow and attention to minimizing additional burden are important considerations in planning implementation. For example, Patton et al. (2020) found that integration of assessments improved implementation of a programme to educate parents of newly diagnosed paediatric cancer patients on what to expect after discharge. Common barriers to successful implementation include lack of engagement or ownership, lack of resources, increased workload of staff and lack of sustainability (Melnyk et al., 2018).

Conducting interviews with key stakeholders can provide insights into implementation processes that produce reliable processes and desired outcomes (Adams, 2015). Successful implementation of interventions in research studies is often judged by quantitative outcomes. However, researchers miss insights from participants about often overlooked contextual information that could contribute to better understanding of the quantitative results (Newcomer et al., 2018).

This study served as the implementation evaluation component of an international, clusterrandomized multi-site clinical trial that used nurse assessment and patient self-assessment of readiness for hospital discharge on the day of discharge as a mechanism to improve discharge preparation with the goal of reducing return to the hospital postdischarge (the READI study [Readiness Evaluation and Discharge Intervention], Weiss et al., 2019). The multi-site study team included four nurse researchers who conducted this implementation evaluation study. Each site was managed by a site principal investigator $(\mathrm{PI})$ assigned by the participating hospitals; each site $\mathrm{PI}$ was partnered with one of the four nurse researchers as a liaison with the multi-site study team. All clinical nurses from the randomly assigned implementation units in 33 Magnet hospitals (1 unit per hospital, 31 US hospitals, 2 Saudi Arabia hospitals) were trained in the study protocol procedures (more than 1,500 nurses study- 
wide); nurses from the control units (1 unit per hospital) performed their usual care discharge practices unaware of the study protocols. Three variations of a discharge readiness assessment protocol were implemented in sequence over the 13-month implementation progressing from nurse assessment only (Protocol 1), to patient and nurse assessments (Protocol 2), to the addition of a requisite to act on low scores (Protocol 3 (described in Table 1) (see Weiss et al., 2019 for more detailed information about the larger study). Unit discharge practices prior to the study varied in their involvement in hospital and unit readmission reduction initiatives, use of readmission risk reduction protocols and configurations of discharge roles (such as unit-based case managers and/or discharge coordinators); none had discharge readiness assessment by either the patient or nurse as part of their discharge protocols (Bobay et al., 2015).

TABLE 1. Study aims and focus group interview guide

\begin{tabular}{|c|c|}
\hline \multicolumn{2}{|l|}{$\begin{array}{l}\text { Goal: to evaluate the implementation } \\
\text { of the READI study protocols from } \\
\text { the perspectives of participating } \\
\text { clinical nurses: }\end{array}$} \\
\hline $\begin{array}{l}\text { Protocol 1: Discharge readiness } \\
\text { assessment by the discharging nurse }\end{array}$ & \\
\hline $\begin{array}{l}\text { Protocol 2: Patient self-assessment of } \\
\text { discharge readiness used to inform } \\
\text { discharging nurse assessment of } \\
\text { discharge readiness }\end{array}$ & \\
\hline \multicolumn{2}{|l|}{$\begin{array}{l}\text { Protocol 3: Patient and nurse } \\
\text { assessment of discharge readiness as } \\
\text { in protocol } 2 \text { plus a requirement for } \\
\text { the discharging nurse to act on all } \\
\text { low readiness scores. }\end{array}$} \\
\hline Implementation evaluation aims & Focus group interview questions \\
\hline \multicolumn{2}{|l|}{$\begin{array}{l}\text { Aim 1: Describe practice changes in } \\
\text { unit and individual nurses' discharge } \\
\text { processes over the course of the } \\
\text { study }\end{array}$} \\
\hline \multirow{2}{*}{$\begin{array}{l}\text { (a) Changes in nursing discharge } \\
\text { practices during the } 13-\text { month } \\
\text { implementation period }\end{array}$} & $\begin{array}{l}\text { 1. How has the discharge process changed on your unit in } \\
\text { the past year? What factors led to these changes? }\end{array}$ \\
\hline & $\begin{array}{l}\text { 2. What is the same, and different, about how you approach } \\
\text { preparing your patient for discharge compared with one } \\
\text { year ago? }\end{array}$ \\
\hline $\begin{array}{l}\text { (b) Discharge readiness assessment } \\
\text { before implementation of the study } \\
\text { protocols }\end{array}$ & $\begin{array}{l}\text { 3. Did you routinely assess for discharge readiness before } \\
\text { the study? If so, how and what did you do with that } \\
\text { information? }\end{array}$ \\
\hline \multirow[t]{2}{*}{$\begin{array}{l}\text { (c) Challenges in implementation of } \\
\text { the study protocols }\end{array}$} & $\begin{array}{l}\text { 4. Describe your experiences with participating in the READI } \\
\text { study. }\end{array}$ \\
\hline & $\begin{array}{l}\text { 5. How difficult was it to complete the discharge readiness } \\
\text { assessment for every patient? }\end{array}$ \\
\hline
\end{tabular}




\begin{tabular}{|l|l|}
\hline $\begin{array}{l}\text { (d) How did practice change from } \\
\text { Protocol } 1 \text { Protocols } 2 \text { and 3? }\end{array}$ & $\begin{array}{l}\text { 6. Do you think adding the patient's perspective to the } \\
\text { assessment (in Protocol 2) changed your assessment from } \\
\text { Protocol 1? How? }\end{array}$ \\
\hline $\begin{array}{l}\text { 7. Did the discharge action guide assist in determining } \\
\text { appropriate interventions in phase 3? }\end{array}$ \\
\hline $\begin{array}{l}\text { 8. Which tools did you find most helpful in assessing } \\
\text { discharge? }\end{array}$ \\
$\begin{array}{l}\text { - Protocol 1: Nurse Assessment, } \\
\text { - Protocol 2: Patient assessment, } \\
\text { for patient discharge }\end{array}$ & $\begin{array}{l}\text { 9. Will you continue to do discharge readiness assessment } \\
\text { on all patients in the future? Would you recommend the } \\
\text { tools be retained in your practice setting: if yes, which ones? }\end{array}$ \\
\hline $\begin{array}{l}\text { Aim 2: Describe nurses' perceptions } \\
\text { about participating in a unit-level } \\
\text { nursing research study }\end{array}$ & $\begin{array}{l}\text { 10. The READI study was a large-scale study, with a long data } \\
\text { collection period that required nurse participation for over } \\
1 \text { year. Talk about your reactions to participating in a study } \\
\text { of this scope. Can you describe what you found most } \\
\text { positive about the experience; What were the negatives? }\end{array}$ \\
\hline $\begin{array}{l}\text { 11. Your hospital was one of more than 30 study sites. Has } \\
\text { your perception of the importance of nursing research } \\
\text { changed as a result of participation in the study? What has } \\
\text { changed? }\end{array}$ \\
\hline
\end{tabular}

\section{METHODS}

\subsection{Design}

This implementation evaluation study used a focused ethnographic approach, a pragmatic and efficient method for investigating the discrete, predetermined phenomena and contexts (Rashid et al., 2015) to identify the shared features and understand the complexities surrounding an issue from the participants' perspectives (Cruz \& Higginbottom, 2013). In implementation research using focused ethnography methods, focus group interviews are a common method for researchers to engage with participants to elicit emic (insider) perspectives and embedded meanings associated with nurses' practice experiences (Bunkenborg et al., 2017; Mekki et al., 2017). In this study, we used focus group interviews as they are well suited for studies exploring attitudes, opinions and experiences in specific groups and contexts (Ravitch \& Carl, 2016), and provide the most efficient method of obtaining staff nurse perspectives due to the number of sites and number of participating nurses.

Human subjects' approval was obtained from the Marquette University Institutional Review Board (IRB) of the PI (M.W.) and the participating hospital IRBs. Approval for the conduct of focus groups was included. Informed consent was obtained from each nurse participating in the focus group by the site PI.

\subsection{Sample and setting}

Ninety-one per cent ( $N=30$ of 33 sites, 28 US and 2 Saudi Arabia hospitals) of study intervention units participated in focus groups. Three sites did not participate due to scheduling issues. Focus groups were scheduled at the end of the quantitative data collection and conducted from March 2016 to 
October 2016. A single focus group was conducted with each site. Site PIs and unit managers notified unit nurses about the focus groups and encouraged participation, which was voluntary, recruiting three to seven nurses to participate in each focus group in keeping with recommendations of focus group sizes not greater than 10 (Speziale \& Carpenter, 2007). A total of 135 nurses participated in the focus groups. The single criteria for focus group participation were use of study protocols. The only information collected about the nurse participants was experience as a nurse, which ranged from 6 months to 44 years. The focus groups were scheduled at a time determined by the unit management team as convenient for the unit's workflow and conducted in a private space near the nursing unit.

\subsection{Data collection}

Each focus group was conducted by two of the nurse researchers, neither of whom were their partner researcher. Focus groups were conducted via audio-conferencing; sessions were audio-recorded. One nurse researcher conducted the interviews while a second nurse researcher took verbatim notes. Participants were instructed to avoid using their own, patient or hospital names during the focus groups; identifying information was not included in verbatim notes. Only de-identified data were reported. Focus group sessions lasted from 30 to 60 min until participants indicated they had no further input.

The focus group format followed a semi-structured interview guide (Table 1). Questions were developed by the research team and were based on eliciting staff nurse perceptions about their practice, practice change and experiences with each of the study protocols. Participants were asked to reflect on study-driven changes, including organisation-driven changes, and to compare their approach to patient discharge preparation at the end of the study versus before implementing the study. Insights were sought into the reasons for continuation or discontinuation of the readiness assessments at the end of the study, nurses' experiences with participating in the study and the value of participating in a large-scale study. Researchers supported elaboration of responses and built on the responses of other participants. Areas of agreement and disagreement were explored. Nurses were encouraged to express both positive and negative aspects of their individual experiences and as a unit.

\subsection{Analysis}

Verbatim notes were the primary data for analysis. Audiotapes of all focus group discussions were available as needed for clarification. Due to potential bias associated with research team members interviewing and taking verbatim notes, an independent researcher (R.C.P.) with expertise in qualitative content analysis who was not part of the original research team, read and independently analysed the verbatim notes using the directed content method of qualitative data analysis (Hsieh \& Shannon, 2005). In directed content analysis, the thematic analysis focused on the a priori structure of the sequential implementation of discharge readiness protocols as outlined through the interview questions, before synthesizing common themes across the entirety of the interviews. Final themes and analysis by the independent researcher were reviewed by the team for validation and interpretation.

\section{RESULTS}

Four themes were identified upon analysis of the focus group narratives. 


\subsection{Nurse awareness}

Most nurses indicated that they became more aware of patient discharge needs and began to think about discharge earlier in the hospitalization. 'Discharge is quicker, patients stay less time, feel like we miss things since it is a short time' and 'never thought about asking these questions' were statements made by several nurses. Nurses reported using the discharge readiness assessment scales as checklists to increase awareness of readiness. Participants noted improvement in nurses' awareness of specific patient questions and concerns, whereas before it was 'just what we knew or we missed things'. They became more aware of psychosocial issues and now involve social services earlier in the discharge process.

\subsection{Changes in nurses' discharge practices}

Many nurses indicated that they did not assess for discharge readiness prior to the study. 'We didn't do it before; we were less aware. We just told patients they were going home'. Nurses reported changing their practice as a result of study participation because they are asking questions now that they did not ask prior to the study. Being cognizant of specific factors when getting patients ready for discharge allowed nurses to make changes based on patient needs. One nurse said:

I did assess before but didn't always focus on specifics. Before I assumed more but didn't really ask. Questions about readiness for home were not as formal before. I focused more on physical needs rather than everything about going home. Since care managers see all patients, I often assumed they asked these questions.

Nurses indicated that the discharge readiness forms provided content and structure for assessment. Nurses in one focus group agreed that nurses were more aware of patient's comfort with going home so changes could be made prior to discharge. A participant reflected on the ease of use of the discharge readiness assessment scales and how they are 'organized to concentrate on patient's needs before going home. It helps us identify if patients are physically and emotionally ready to go home and helps identify special needs for patients going home that need to be arranged'.

Several nurses mentioned how the new approach to discharge helped new nurses identify patient needs and provided a checklist (form) for them to follow. For new nurses, the RN assessment form introduced them to the expected practice of discharge readiness assessment. One RN who joined the unit after the study began said, 'this is the only way I have done discharge, so it has built my practice'.

At several sites, nurses reported that the approach to preparing patients for discharge did not change. Among nurses who did not report a change in how they prepared patients, one participant said, 'It is not really different than what we were doing. We always checked to see if patients were safe to go home and had help at home'. One nurse questioned the value of discharge readiness assessments given other discharge roles stating, 'I thought about it more but didn't change my actions; we have discharge planners and they are awesome'. An additional participant explained it differently, '[The discharge] process didn't change but nurses [are] looking at it differently'.

Nurses were asked if they thought adding the patient's perspective (Protocol 2) to the assessment changed the nurse assessment. Responses indicated that the patients' perspective may or may not change the nurses' original assessment, though the nurses reported that in many cases, the nurses' 
assessments were congruent with those of the patients and reinforced the nurses' assessments. One nurse stated that the patient's form was most helpful 'when there was a difference' between the nurse and patient rating. Several participants reported that adding patient's perspective was positive and beneficial because it allowed patients the opportunity to be involved in their care. Patients were in general receptive to participating, with few patients reluctant or refusing to participate.

There were several nurses who indicated that the patient's perspective did impact the nurses' assessment. The nurses said it offered an 'opportunity to step back and see if there is something we needed to do' and 'makes you investigate further, is there something else I can do?' One nurse said, 'Phase [protocol] 2 enlightened us in knowing how ready patient was'. Others pointed out that Protocol 2 allowed further opportunity to educate patients and facilitated more collaboration with the discharging physician and social work. Several nurses stated that including the patient's perspective was a positive practice change for them leading to further discussions about social situations and daily life, which had not been previously considered. One participant did not find any of the forms to be helpful.

Nurses were asked if a discharge action guide used in Protocol 3 to help nurses to identify actions to take in response to low discharge readiness assessments assisted in determining appropriate interventions. Several nurses indicated that the guide did not assist because many of the determinations were made prior to the discharging nurse's involvement. Patients with low discharge readiness score were often referred to a case manager or nurse navigator. Experienced nurses found the guide less useful than newer nurses; however, one nurse reported it helped them to 'catch what we missed'. Newer nurses 'needed a little push to know what to do'.

\subsection{Patient and family involvement in the discharge process}

The discharge readiness assessments were viewed by most nurses as a positive initiative that improved communication and coordination among disciplines and with patients and families. The emphasis on patient involvement, planning for the patient discharge earlier in the hospitalization, increased patient education, assuring patient's readiness for discharge and giving patients a voice was viewed as a beneficial practice change. One nurse observed, 'Many changes are for the better; we are more concerned about the patient and family; there is more planning ahead for discharge and more consideration of individual needs'. Another nurse noted, 'the new process involves the caregivers more, whereas before they only involved the patient. Now they ask if someone is at home and concentrate more on the caregivers'. The protocol allowed the nurses to incorporate patient preferences into discharge planning. In contrast, nurses also commented on increased difficulty when trying to discharge patients after getting the patient perspective. Sometimes this had to do with language or comprehension issues or it added workload when nurses noted problems that needed to be resolved prior to discharge.

\subsection{Synergy and enhancement of discharge processes}

A central theme raised by nurses was that the introduction of discharge assessment protocols integrated with and enhanced existing processes. Specifically, the protocols facilitated early identification of needs for transition to home and supported discharging patients in a timely manner. 
Several nurses mentioned the need to meet specific timelines for discharges and that the new process helped reach their goal of 'getting better at getting patients out earlier'.

The addition of the discharge readiness protocols helped to formalize the process. One participant reported that they now had a 'more formal way of assessing that patients are ready to go'. One nurse said, 'Now they have an idea of how discharging patients should be'.

Changes in the discharge process also facilitated better care coordination between pharmacy, social workers, physicians, case managers and nurses. One nurse indicated that the readiness assessment protocols improved discussions with MDs for patients not ready to go home. Before (the study), it was whatever the doctor says'. Several participants stated that this experience increased their comfort with discussing patients with physicians and other team members. Because discharge planning was often done by case managers, discharge planners or care coordinators, they made assumptions that these other team members were assessing the patients for readiness to go home, but that there was no formal process in place before the study. Communication about discharge readiness assessments was not uniformly valued; not all physicians were receptive to being told that a patient was not ready to go home.

\subsection{Implementation challenges}

Challenges reflected the realities of integrating new practices into routine care. Many nurses commented on the difficulty of completing the discharge readiness assessment for every patient, noting that they would sometimes forget to complete the assessments on a busy day or after days off. The added time for assessment and documentation was cited as a burden interfering with fidelity for completing the protocols. A nurse summed up both concerns: 'It is more difficult to remember than to do the actual form. Nurses are a bit overwhelmed with more duties, extra time'. It took more time and was more difficult to complete the forms with certain types of patients, such as nonadherent patients and patients in pain. Time burden was particularly an issue with patients who had low literacy or were non-English speaking. One nurse stated: 'I routinely needed to explain some questions to the patients because they didn't understand'. Completing the discharge readiness assessment forms was also perceived to be an added burden for family members who sometimes helped with reading and answering the readiness assessment questions when the patient could not fully comprehend.

The additional work imposed by the study protocols evoked both negative reactions and creative responses. 'They are always adding another step for nurses' and 'It's just another piece of paper we had to fill out'. To address this issue, making the study a unit project and including the unit secretaries in the process was among the proactive solutions: 'It took a whole floor effort to complete. They reminded each other. Unit secretary was on top of it'. A particular concern noted by the nurses was the use of paper forms for discharge readiness assessment in an era of paperless records; they wished the forms were incorporated into the EHR.

Validity of the assessments emerged as a concern among a small number of nurses. One nurse was concerned with the Protocol 3 in which nurses were informed of a specific cut-off score on the assessments that indicated low readiness: 'Sometimes knowing you had to do something if you scored [a low readiness score] was limiting; there was a fear that we had to do something, so we may have inflated scores'. Others expressed concerns about patient honesty suggesting that patients wanting to 
go home would rate themselves high 'just to get out' and patients not wanting to go home would rate themselves low to stay hospitalized. One nurse said, 'Some patients felt they were being quizzed and they were trying to give the right answer. They were afraid they might not get discharged or would get into trouble'.

The protocols were implemented for effectiveness testing. Nurses at many sites stated they would continue to use the discharge readiness assessment in the future, and recommended integration into the EHR. Another recommendation was to include the discharge readiness assessment tools in new nurse orientation. Sites not continuing use of the assessment stated that they had future plans for EHR conversions or updates and could not build the forms into the existing EHR, they were already using similar tools, or that with a care coordination team in place, readiness assessments were redundant.

\subsection{Participation in nursing research}

Many nurses indicated that their perceptions about nursing research were more positive and participation in the study helped them understand the research process better. For many, this was their first opportunity to participate in research. Interestingly, those who stated their perceptions had not changed went on to comment on the positive impact the research had on patient care. Only one nurse said, 'I don't see benefit in participating'. Other nurses who were currently pursuing graduate education commented that it made the research process real.

Personal and professional pride in being part of research, and especially an international multi-site study, was expressed as 'It's nice to know that things can change and that I can be part of that change which may positively affect the future' and 'it is great to hear the voice of the nurse and potential changes came from nurses'. Many of the nurses expressed the benefit of research for patient care through 'improv[ing] nursing practice'. Several nurses expressed a desire to be involved in more research. One nurse said, 'I sit on a team that is looking at readmission so was excited to have the study come along. I can see how important these questions are and how this impacts the hospital'. Another said, 'I think nursing research is necessary, [it] gives us nurses a voice in what we think about the patients and their readiness for discharge'. In many cases, nurses were included in the decision by their managers to volunteer their unit for the study. When nurses were not included in the decision, 'we were told what to do, so didn't feel empowered by study, stating they felt the assessment protocol was 'just a task', or that we are 'worker ants'.

\section{DISCUSSION}

When describing how participation influenced how nurses perform discharge-related processes, the primary emerging theme was increased and earlier awareness and attention to discharge needs. Nurses reported changes to their discharge practices, patient and family involvement in discharge processes, synergy and enhancement of discharge processes, and challenges to implementation of structured discharge readiness assessments. Finally, in describing nurses' perceptions about participating in a multi-site, unit-level, nursing implementation research study, the overwhelming responses were positive with nurses expressing pride in participating in research and having a voice in the discharge process for patients. Similar responses have been reported from other multi-site studies (Friese et al., 2017; Patton et al., 2020). Friese et al. (2017) noted that engaging nurses in how they 
wanted to learn the content was key. Patton et al. (2020) reported that clinical nurses found that being part of a multi-site study increased their engagement in the research process.

Incorporating patients in the discharge process benefitted nurses and their patients. It contributed to a change in nursing practice by providing a way to consider the patient's perspective and incorporate patient and family input. This process also allowed nurses to provide additional education, arrange additional resources, prepare the patient and family for discharge home and avoid readmissions. In the quantitative analysis for the study, adding the patient's perspective to the nurse's discharge readiness assessment resulted in a reduction in readmissions in patients from high-readmission units (Weiss et al., 2019).

Conducting clinical nurse focus groups helped to clarify quantitative results from the study. Triangulation of qualitative and quantitative results is useful in implementation studies to better understand context as it affects acceptability, adoption and sustainability of the intervention (Hamilton \& Finley, 2019). The research team was able to identify possible alternative explanations for increased mean readiness scores in Protocol 3 (Weiss et al., 2019). For example, nurses confirmed that they sometimes 'over-coded' the readiness scores so that they did not have to document additional interventions to address low scores.

One of the most exciting findings was the consistent message from nurses about how their practice changed as a result of participating in the translational research study. Nurses could see how the change in their practice could lead to better patient care and outcomes. Participating in a unit-based research study made nursing research real, not conceptual, for clinical nurses. Research that is relevant to nursing practice not only carries more meaning for nurses but contributes to increased participation (Westerlund, Nilsen, \& Sundberg, 2019). When presented with strong evidence that the proposed practice change will improve patient care and results are visible, the likelihood of adoption of an innovation increases (Mathieson et al., 2018).

In the course of the focus groups, nurses provided important context for considering future implementation efforts. For example, they raised questions about the many health team members involved in the discharge process and who among them should be conducting discharge readiness assessments. Future studies will need to assess the relative value of the discharging nurse conducting the assessment or another member of the discharge team such as the discharge coordinator or case manager. Of note, we found in another of our studies that patients who had continuity of care from their discharging nurse (when the patient received care from the same nurse on the day of and day before discharge) had lower readmission rates than those without this continuity (Bahr et al., 2020).

Strengths of this study include the large number of nurse participants in the focus groups, the number of sites represented and the use semi-structured interview questions to direct attention to evaluation of the context and processes of implementation of the study. A trained qualitative nurse researcher led the analysis to reduce bias in analysis. Limitations include the use of Magnet hospitals as study sites; these hospitals have a requirement for engagement in nursing research. Only one focus group was conducted per site. The nurse researchers conducting the interviews were known to the study sites, having worked with them throughout the study in support of study operations. Nurses participating in the focus groups may have answered in a way to please the researchers, although the analysis 
indicates that nurses felt free to share both positive and negative thoughts. While clinical nurses were forthcoming in responses, it is possible that they did not reflect the full range of views of their nursing unit colleagues. Another possible limitation was the method of conducting focus groups by audioconferencing, not in person or face to face. Lastly, focus groups were only conducted on the study implementation units, precluding any comparisons of nurse perceptions of the discharge processes under usual care conditions.

\section{CONCLUSION}

Participation in a unit-level translational research project was a successful strategy for engaging nurses in practice change to improve hospital discharge. Nurses reported improved and earlier awareness of patients' discharge needs, changes in discharge practices, greater patient/family involvement in discharge, synergy and enhanced discharge processes, and implementation challenges. Participating nurses related the benefits of participation in nursing research. Participating in nursing research that has a direct impact on practice is appealing to clinical nurses, improving their perceptions of the relevance of research and providing a sense of professional pride in affecting nursing care practices.

\section{IMPLICATIONS FOR NURSING MANAGEMENT}

Leading unit-based implementation of a structured discharge readiness assessment brings benefits for patients, nurses and the health care team. Including nurse assessment and patient self-assessment encourage earlier awareness of patients' discharge needs, improved patient assessment and greater patient/family involvement in discharge preparation. Integrating discharge readiness assessments into existing discharge care promotes communication between health team members that facilitates a timely, coordinated discharge.

Participating in translational nursing research provides clinical nurses the opportunity to engage in testing new interventions for their effectiveness with patients and for the feasibility of implementing as new standard care practices. For nurse managers, encouraging their clinical nurses to engage in translational research that tests new nursing practices within the real-world context of clinical practice can bring about enhanced nursing practices, improved patient outcomes and excitement about the processes of creating practice change. Ownership of the practice change in creating positive change in practice and outcomes enhances nurse satisfaction and professional identity (Liu et al., 2016).

Building a culture of innovation in care practices through research that translates evidence-based practices to implementation requires commitment from nursing leadership. Unit-based implementation evaluation is a mechanism for involving nurses in clinical nursing research that directly affects their practice. It can increase awareness of routine care processes, such as discharge assessment, that can be improved through implementation and evaluation of evidence-based protocols. Nurses may develop an enhanced sense of their role as a result of elevating practice.

The evidence from this study confirms recommendations for implementation of assessment of discharge readiness as a standard practice for hospital discharge (Weiss et al., 2011, 2014), yet this practice is still not widespread, nor incorporated into assessments in EHRs. In this study, use of structured discharge readiness assessment prompted nurses to obtain additional information from patients and families to encourage more successful discharge. Nurse managers may consider working 
with their unit staff to implement discharge readiness assessment as a standard nursing practice within their local context.

Lessons learned from the implementation process will be useful to nurse managers in planning future translational research efforts. For example, nurses appreciated that the discharge readiness assessments used structured forms that were short in length to minimize the time burden. They would have preferred the forms be loaded into the EHR since many no longer used any paper forms.

Integration of research data collection within the EHR is an important innovation to move translational research forward at a more rapid pace. Another lesson learned is that future projects involving unitlevel implementation consider the complexity of the many roles involved in discharge and how best to integrate the discharge readiness assessment process for optimal patient input and outcome as well as clinician processes.

Support from all levels of nursing management from Chief Nurse Executive to unit management teams is critical for implementation of new practice protocols. The multi-level engagement of executive and unit-level management was critical to the successful implementation of the discharge readiness assessment protocols over the many months of the READI study (Costa et al., 2020). Recognition from nursing leadership for the clinical nurse efforts towards successful implementation was a key factor in achieving high fidelity to the intervention (over $70 \%$ of patients received the new discharge assessment protocols (Weiss et al., 2019). Nurses in the focus groups reflected on their role in decisions about participation in the study; nurses valued being included in the decision and were less enthusiastic when a top-down decision was presented to them. Planning for high-level involvement of unit and hospital leadership during preparation for a translational research project to introduce and evaluate new practice protocols will contribute to successful implementation and sustainability at the end of the study.

\section{ACKNOWLEDGEMENTS}

The authors would like to thank all onsite principal investigators and the nurses who participated in the focus groups.

\section{CONFLICT OF INTEREST}

This study was commissioned by the American Nurses Credentialing Center (ANCC) through a competitive application process (Weiss, PI). The study was funded through participation fees from study hospitals. The research team and the results, analysis, conclusions and recommendations of the study are independent of ANCC and do not necessarily reflect the views of ANCC.

\section{AUTHOR CONTRIBUTIONS}

$\mathrm{MW}, \mathrm{KB}, \mathrm{RH}$ and $\mathrm{LC}$ designed the study. MW, KB, RH, LC, SB, DS and SN collected data. KB, MW and $\mathrm{RCP}$ analysed the data. $\mathrm{KB}, \mathrm{MW}$ and RCP prepared the manuscript. All authors approved the final version for submission. 


\section{ETHICAL APPROVAL}

Human subjects' approval was obtained from the Marquette University Institutional Review Board (IRB) of the principal investigator (Weiss, \#2668) and the participating hospital IRBs.

\section{REFERENCES}

Adams, W. C. (2015). Conducting semi-structured interviews. In K. E. Newcomer, H. P. Hatry, \& J. S. Wholey (Eds.), Handbook of practical program evaluation ( 4th ed.). Wiley \& Sons.

Bahr, S. J., Bang, J., Yakusheva, O., Bobay, K. L., Krejci, J., Costa, L., Hughes, R. G., Hamilton, M., Siclovan, D. M., \& Weiss, M. E. (2020). Nurse continuity at discharge and return to hospital. Nursing Research, 69(3), 186- 196. https://doi.org/10.1097/NNR.0000000000000417

Bobay, K., Bahr, S. J., Weiss, M. E., Hughes, R., \& Costa, L. (2015). Models of discharge care in Magnet hospitals. Journal of Nursing

Administration, 45(10), 485- 491. https://doi.org/10.1097/NNA.0000000000000239

Bunkenborg, G., Bitsch Hansen, T., \& Hølge-Hazelton, B. (2017). Handing over patients from the ICU to the general ward: A focused ethnographical study of nurses' communication practice. Journal of Advanced Nursing, 73, 3090-3101. https://doi.org/10.1111/jan.13377

Costa, L. L., Bobay, K., Hughes, R., Bahr, S. J., Siclovan, D., Nuccio, S., \& Weiss, M. (2020). Using the consolidated framework for implementation research to evaluate clinical trials: An example from multisite nursing research. Nursing Outlook.

Cruz, E. V., \& Higginbottom, G. (2013). The use of focused ethnography in nursing research. Nurse Researcher, 20(4), 36- 43. https://doi.org/10.7748/nr2013.03.20.4.36.e305.

Friese, C. R., Mendelsohn-Victor, K., Ginex, P., McMahon, C. M., Fauer, A. J., \& McCullagh, M. C. (2017). Lessons learned from a practice-based, multi-site intervention study with nurse participants. Journal of Nursing Scholarship, 49(2), 194- 201. https://doi.org/10.1111/jnu.12279

Glasgow, R. E., Kessler, R. S., Ory, M. G., Roby, D., Gorin, S. S., \& Krist, A. (2014). Conducting rapid, relevant research: Lessons learned from the My Own Health Report Project. American Journal of Preventive Medicine, 47(2), 212- 219. https://doi.org/10.1016/j.amepre.2014.03.007

Hamilton, A. B., \& Finley, E. P. (2019). Qualitative methods in implementation research: an introduction. Psychiatry Research, 280, 112516.

Hsieh, H.-F., \& Shannon, S. E. (2005). Three approaches to qualitative content analysis. Qualitative Health Research, 15(9), 1277-1288. https://doi.org/10.1177/1049732305276687

Liu, Y., Aungsuroch, Y., \& Yunibhand, J. (2016). Job satisfaction in nursing: A concept analysis study. International Nursing Review, 63(1), 84-91. https://doi.org/10.1111/inr.12215

Mathieson, A., Grande, G., \& Luker, K. (2018). Strategies, facilitators, and barriers to implementation of evidence-based practice in community nursing: A systematic mixed-studies review and qualitative synthesis. Primary Health Care Research \& Development, 20(e6), 1- 11. https://doi.org/10.1017/S1463423618000488

Mekki, T. E., Øye, C., Kristensen, B., Dahl, H., Haaland, A., Nordin, K. A., Strandos, M., Terum, T. M., Ydsteb $\varnothing$, A. E., \& McCormack, B. (2017). The inter-play between facilitation and context in the promoting action on research implementation in health services framework: A qualitative exploratory implementation study embedded in a cluster randomized controlled trial to reduce restraint in nursing homes. Journal of Advanced Nursing, 73, 2622- 2632. https://doi.org/10.1111/jan.13340 
Melnyk, H., Rosenfeld, P., \& Glassman, K. S. (2018). Participating in a multi-site study exploring operational failures encountered by frontline nurses. Journal of Nursing Administration, 48(4), 203- 208. https://doi.org/10.1097/NNA.0000000000000600

Munro, C. L., \& Savel, R. H. (2016). Narrowing the 17-year research to practice gap. American Journal of Critical Care, 25(3), 194- 196. https://doi.org/10.4037/ajcc2016449

K. E. Newcomer, H. P. Hatry, \& J. S. Wholey (Eds) (2018). Handbook of practical program evaluation ( 4 th ed.). Wiley \& Sons.

Patton, L., Montgomery, K., Coyne, K., Slaven, A., Arthur, M., \& Hockenberry, M. (2020). Promoting direct care nurse engagement in research in Magnet hospitals. Journal of Nursing Administration, 50(5), 287- 292. https://doi.org/10.1097/NNA.0000000000000885

Rabin, B. A., \& Brownson, R. C. (2018). Terminology for dissemination and implementation research. In R. C. Brownson, G. A. Colditz, \& E. K. Proctor (Eds.), Dissemination and implementation research in health: Translating science to practice ( 2 nd ed.). Oxford University Press.

Rashid, M., Caine, V., \& Goez, H. (2015). The encounters and challenges of ethnography as a methodology in health research. International Journal of Qualitative Methods, 2015, 1- 16. https://doi.org/10.1177/1609406915621421

Ravitch, S. M., \& Carl, N. M. (2016). Qualitative research: Bridging the conceptual, theoretical, \& methodological. Sage.

Speziale, H. J. S., \& Carpenter, D. R. (2007). Action research method. Qualitative Research in Nursing: advancing the humanistic imperative, 327- 348.

Titler, M. G. (2018). Translation research in practice: An introduction. Online Journal of Issues in Nursing, 23(2), 1. https://doi.org/10.3912/OJIN.Vol23No02Man01

Weiss, M. E., Bobay, K. L., Johantgen, M., \& Shirey, M. R. (2018). Aligning evidence-based practice with translational research: Opportunities for clinical practice research. Journal of Nursing Administration, 48(9), 425- 431. https://doi.org/10.1097/NNA.0000000000000644

Weiss, M. E., Costa, L. L., Yakusheva, O., \& Bobay, K. L. (2014). Validation of patient and nurse short forms of the Readiness for Hospital Discharge Scale and their relationship to return to the hospital. Health Services Research, 49(1), 304- 317. https://doi.org/10.1111/1475-6773.12092

Weiss, M. E., Yakusheva, O., \& Bobay, K. L. (2011). Quality and cost analysis of nurse staffing, discharge preparation, and postdischarge utilization. Health Services Research, 46(5), 1473- 1494. https://doi.org/10.1111/j.1475-6773.2011.01267.x

Weiss, M. E., Yakusheva, O., Bobay, K. L., Costa, L., Hughes, R. G., Nuccio, S., Hamilton, M., Bahr, S., Siclovan, D., \& Bang, J. (2019). Effect of implementing discharge readiness assessment in adult medical-surgical units on 30-day return to hospital: The READI randomized clinical trial. JAMA Network Open, 2(1), e187387. https://doi.org/10.1001/jamanetworkopen.2018.7387

Westerlund, A., Nilsen, P., \& Sundberg, L. (2019). Implementation of implementation science knowledge: the research-practice gap paradox. Worldviews on Evidence-Based Nursing, 16(5), 332. 